\title{
Retromandibular Approach as a Method for Open Reduction and Internal Fixation of Mandibular Condyle Fractures. Experience in the Regional Hospital $1^{\circ}$ de Octubre, ISSSTE and Literature Review
}

\author{
Mauricio González Rebattú y González, ${ }^{1, *}$, Ana María Nieto Munguía², Christian Adolfo San Juan González ${ }^{3}$, Elizabeth Balderas \\ Tellez ${ }^{4}$, and Isaí Daniel García Montes ${ }^{5}$
}

\author{
${ }^{1}$ Assigned Physician of the Maxillofacial Surgery Service, H.R. $1^{\circ}$ de Octubre, ISSSTE, Mexico \\ ${ }^{2}$ Assigned Physician of the Maxillofacial Surgery Service, H.R. Lic. Adolfo Lopez Mateos ISSSTE, Mexico \\ ${ }^{3}$ Former resident of the Maxillofacial Surgery Service, H.R. $1^{\circ}$ de Octubre, ISSSTE, Mexico \\ ${ }^{4}$ Fourth year resident of the Maxillofacial Surgery Service, H.R. $1^{\circ}$ de Octubre, ISSSTE, Mexico \\ ${ }^{5}$ Second-year Resident of the Maxillofacial Surgery Service, H.R. $1^{\circ}$ de Octubre, ISSSTE, Mexico
}

*Corresponding author: Mauricio González Rebattú y González, Assigned Physician of the Maxillofacial Surgery Service, H.R. $1^{\circ}$ de Octubre, ISSSTE, Av. National Polytechnic Institute No. 1669, Col. Magdalena de las Salinas, Deleg. Gustavo A. Madero, Mexico, E-mail: mauriciorebattu@ yahoo.com.mx

Received: 05 Jun, 2021 | Accepted: 26 Jul, 2021 | Published: 03 Aug, 2021

Citation: González MGR, Munguía AMN, González CASJ, Tellez EB, Montes IDG (2021) Retromandibular Approach as a Method for Open Reduction and Internal Fixation of Mandibular Condyle Fractures. Experience in the Regional Hospital $1^{\circ}$ de Octubre, ISSSTE and Literature Review. Int J Dent Oral Health 7(5): dx.doi.org/10.16966/2378-7090.376

Copyright: (C) 2021 González MGR, et al. This is an open-access article distributed under the terms of the Creative Commons Attribution License, which permits unrestricted use, distribution, and reproduction in any medium, provided the original author and source are credited.

\begin{abstract}
Mandibular condyle fractures constitute $17.5 \%$ to $52 \%$ of mandibular fractures. Open reduction with internal fixation and closed reduction are described for their treatment. Fonseca describes the absolute indications for open treatment when there is displacement of the mandibular condyle to the middle cranial fossa, foreign body invasion, extra capsular displacement of the condyle, and/or malocclusion not susceptible to closed reduction. It includes indications with vast evidence for open treatment of bicondyle fractures, condylar displacement greater than 45 degrees, reduction in the height of the mandibular ramus greater than or equal to $2 \mathrm{~mm}$, fractures associated with fractures of the middle third and when there is unstable occlusion. The above establishes sufficient evidence to perform open treatment in various situations of condylar fractures. Regarding the approach of the mandibular condyle region there are different surgical techniques, such as, the preauricular, retroauricular, submandibular, retromandibular, and rhytidectomy approaches.
\end{abstract}

In our experience, the retromandibular approach provides adequate visualization for the reduction and fixation of mandibular condyle fractures.

Keywords: Subcondyleal fracture; Retromandibular approach; Transparotideal approach; Mandibular marginal facial nerve

\section{Introduction}

The approach to the mandibular condyle region is complex due to the existence of the branches of the facial nerve which is why the management of condylar fractures comes into discussion as to when to perform an open or closed treatment. Although the absolute and relative indications are well known, the best treatment for each patient continues to be the surgeon's choice $[1,2]$.

Regarding open treatment, different approaches have been proposed; the retromandibular approach described by Hinds in 1967 suggests that a subcondylar mandibular vertical osteotomy in the correction of prognathism is performed. This technique describes the incision below the ear lobe with an incision extension no greater than $2.5 \mathrm{~cm} \mathrm{[3].} \mathrm{Ellis} \mathrm{indicates} \mathrm{that} \mathrm{the} \mathrm{incision} \mathrm{is} \mathrm{made} 0.5 \mathrm{~cm}$ below the ear lobe with an extension of up to $3.5 \mathrm{~cm}$ of length behind the edge of the mandibular ramus and may extend below the level of the mandibular angle [4].

In addition to the trajectory of the facial nerve and its ramifications, important blood vessels are found; we summarize different anatomical references that should be taken into account during the approach to the region of the mandibular condyle.

\section{Suggested Surgical Technique}

In the Maxillofacial Surgery Service of the $1^{\circ}$ de Octubre Regional Hospital, we have chosen to perform a transparotid retromandibular approach, which we describe below including the author's suggestions:

Using general anesthesia with nasotracheal intubation, the surgical area is delimited keeping the corner of the mouth and the ear lobe visible on the side of the fracture. 
1. Marking: starting immediately from the origin of the earlobe, it continues on the posterior border of the mandibular ramus with an extension no greater than $3 \mathrm{~cm}$ (Figure 1).

2. Anesthetic is infiltrated superficially along with $1 \mathrm{cc}$ of vasoconstrictor for hemostatic purposes.

3. Incision and dissection: Subsequently the latency period begins the incision immediately from the origin of the earlobe with a 15 Scalpel blade cutting skin and subcutaneous cellular tissue using blunt Metzenbaum Scissors; a blunt dissection is performed on the platysma muscle to free the skin itself that will be withdrawn to access the desired surgical site. Blunt dissection is continued with the use of Adson Forceps and Metzenbaum Scissors, cutting through the center of the platysma muscle, dissecting in the direction of the initial marking, and, posteriorly, clamping the dissected tissue to avoid injury to the mandibular marginal branch. In spite of the marginal nerve being described as located under the platysma muscle, it is advisable to perform tests to avoid damaging collaterals; once the platysma muscle has been dissected, we will find part of the superficial muscle aponeurotic system which will be dissected with the same technique described above. In the following plane, we will find the superficial lobe of the parotid gland where a transparotid technique is performed. We shall continue dissecting until bone tissue is found, and the fracture line is identified.

4. Reduction: To carry out the reduction of subcondylar fractures, different techniques have been described that allow the mandibular ramus to be displaced caudally. We suggest the use of a McKesson gag, which is placed in the most posterior region of the fractured side to descend the mandibular ramus producing the reposition of the mandibular condyle within the glenoid cavity when the ideal position of the condyle is achieved; we proceed to perform the fixation with the use of condyle plates. The author suggests the use of a trapezoidal plate with four holes; the mouth gag is removed and the mandibular function is verified; the occlusion is corroborated and the closure of the wound by planes is continued emphasizing the closure of the parotid capsule to avoid fistulas. For the skin closure, a subdermal suture is performed. (Figures 2-4)

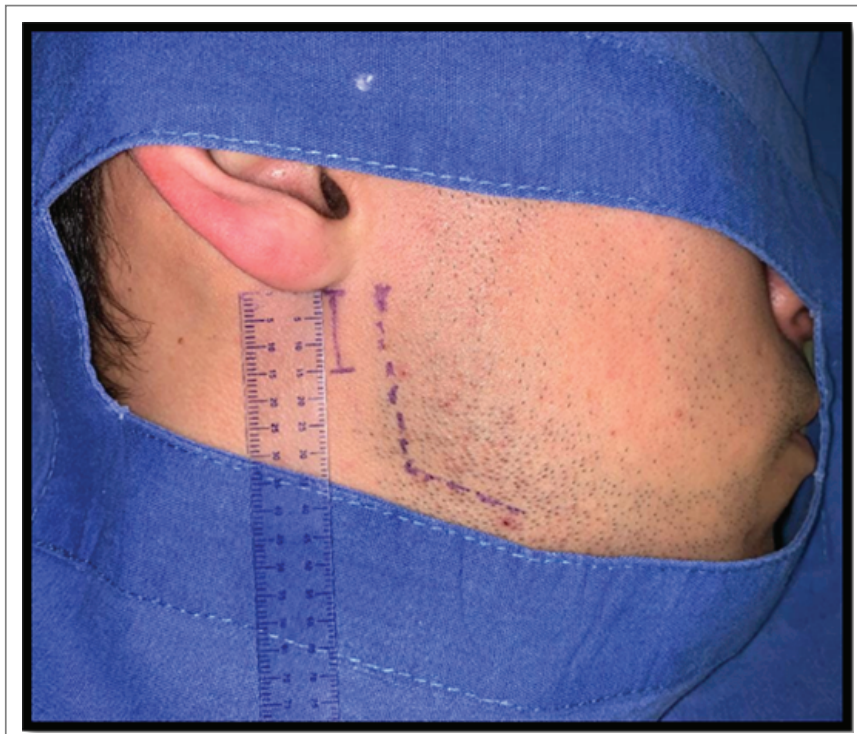

Figure 1: Surgical marking prior to incision, starting from immediately the origin of the ear lobe, it continues on the posterior border of the mandibular ramus with an extension no greater than $3 \mathrm{~cm}$.
In the author's experience for the management of fractures that involve some other mandibular region, it is recommended to begin with the condylar fracture due to the displacement of the mandibular ramus that is required for the replacement of the condyle.

\section{Discussion}

Of the approaches described to reach the mandibular condyle region, the preauricular approach would be the closest approach, however, to avoid injury to the facial nerve, the extension of the approach limits access to the area, whereas the retromandibular approach provides us with an excellent visualization of the condylar area in addition to offering protection to the main branches of the facial nerve, minimizing it by a significant percentage with the sole exception of possible damage to the mandibular marginal branch.

Several authors have described the advantages of using a retromandibular approach compared to other approaches that were used more frequently in open reduction of condylar fractures.

Flores JR, et al., ensures superior advantages to the preauricular approach that, although it provides a good exposure of the condyle and subcondylar regions, produces an excessive scar, specially for young patients regarding safety of nerve structures. In the study he carried out on 31 patients, $5.88 \%$ reported facial paresis, $17.65 \%$ salivary fistula, and $8.82 \%$ postoperative infection, in addition to a vascular necrosis of the condyle and in two cases there was a fracture of the osteosynthesis material [5]. In our experience at the closure of the wound by planes we put emphasis on the closure of the parotid capsule to avoid salivary fistulas and we recommend performing

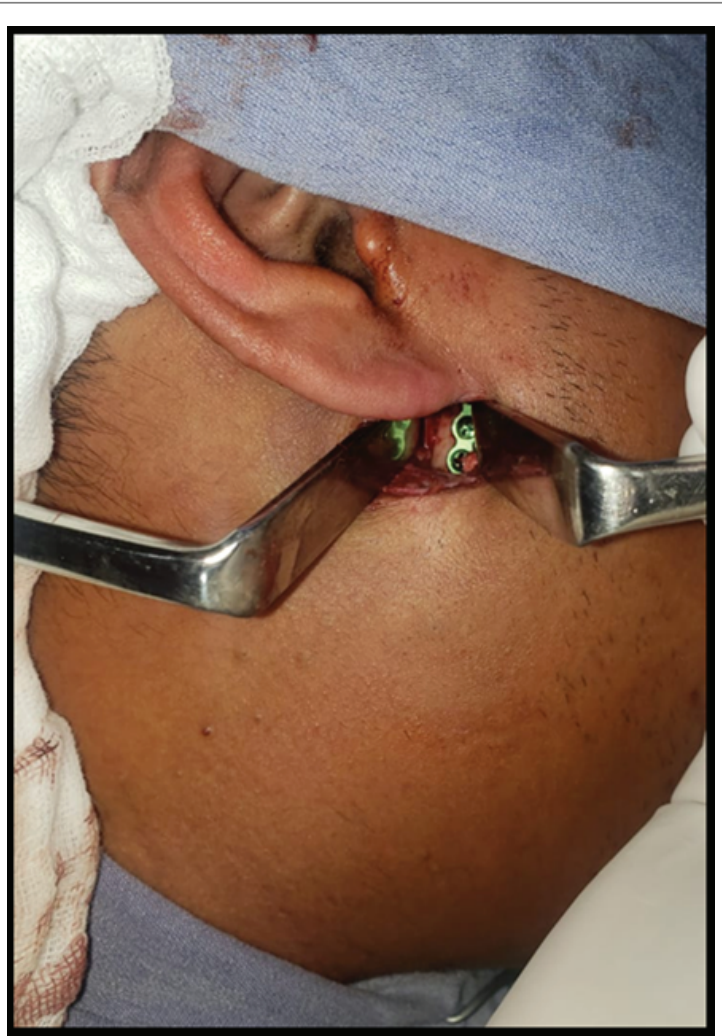

Figure 2: Osteosynthesis material after its placement, where a correct visualization is observed without amplitude greater than $3 \mathrm{~cm}$ in length and a correct parallelism for the placement of the osteosynthesis material. 


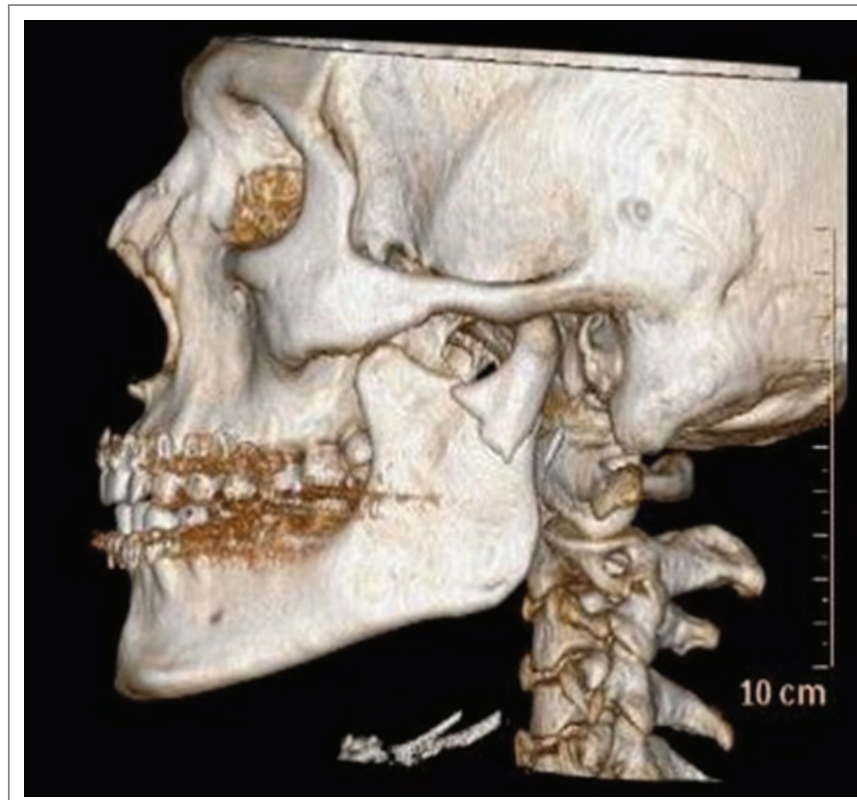

Figure 3: Three-dimensional reconstruction showing a solution of continuity in the condyle on the left side. nerve tests to avoid damaging collateral branches of the facial nerve. Likewise, it ensures that the anterior transparotid approach is an aesthetic approach, due to the incision of just $2 \mathrm{~cm}$ in length causing a discreet scar in an inconspicuous area, in addition to speaking of the technique it allows a vertical placement of the osteosynthesis screws which is crucial in stability, with respect to safety with the facial nerve ensures that the incision is located between the buccal branch and the marginal branch, in addition to that if it is crossed in the field. It can be preserved without damage by blunt dissection in the parotid and muscular planes. At the end of this study, he concluded that the anterior transparotid approach is a safe approach to subcondylar fractures with a low risk of damaging the facial nerve, in addition to providing good exposure to the fracture line that allows the placement of a second plate in the anterior area. Of the same and leaving more acceptable aesthetic results.

It is important to take into account the surgical time being also one of the advantages obtained in this type of approach, this question is described by Bhutia $\mathrm{O}$, et al. In addition to evaluating the affections to the facial nerve, it also describes among its results that the access and exposure of the fracture was 30 minutes and in medially dislocated fractures it was a little more than 30 minutes, which is a benefit not only in postoperative recovery but also in the preservation of condylar vascularity [6]. Regarding the affection of the facial nerve, I observed that $20 \%$ had a transitory paresis affecting the buccal branch in 7 cases, mandibular marginal in 2 cases and zygomatic in 1 case, this compared with others studies such as the one carried out by Yang $L$, et al., in 2012, in which very similar results were reported, observing paresis in $18 \%$ of which the buccal and/or zygomatic branches were affected [7].

In addition to nerve injury, the result of open and closed treatment has been compared, finding that reduction and rigid fixation produces superior anatomy with results with faster restoration of function and shape [8].

The literature describes an intraoral approach with a lower risk of nerve injury; however, its performance requires specialized equipment and involves a longer surgical time [9]. The retromandibular approach

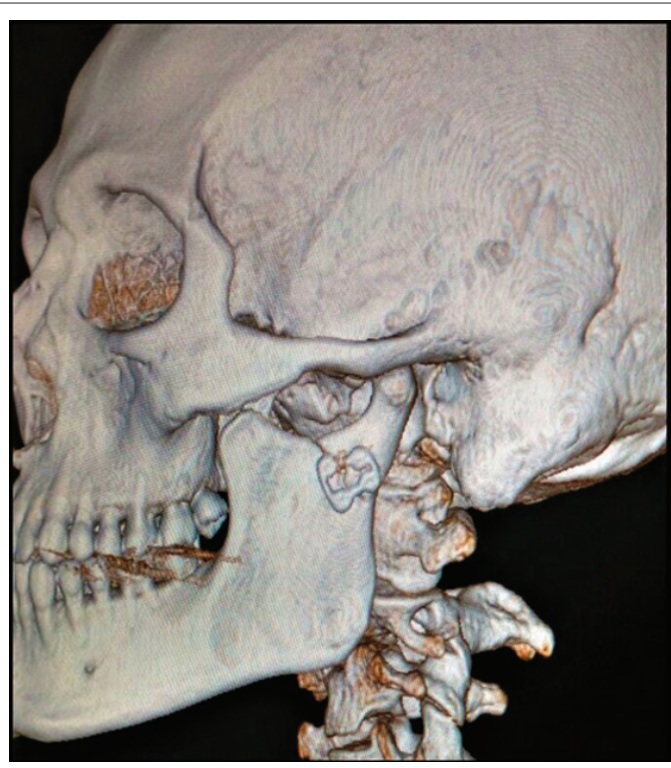

Figure 4: Three-dimensional reconstruction after the placement of osteosynthesis material where adequate reduction of the fragments is observed.

presents variants in its performance; we find the transparotid, retroparotid and also transmaseterine routes. Several studies have been carried out to compare the injuries that each one can cause, evaluating nervous affection, inadequate reduction and presence of parotid fistula, each author reporting discretely different results, however, numerous modifications are presented in the techniques described, however, we suggest that the incision be made immediately below the ear lobe, which avoids a noticeable scar and reduces the risk of injury to the branches of the facial nerve, as well as providing a better visualization of the surgical field [1].

\section{Conclusions}

After reviewing the various studies of the different approaches, we can conclude that the retromandibular approach is an excellent option for open reduction and internal fixation of condylar and subcondylar fractures. At the ISSSTE Regional Hospital $1^{\circ}$ de Octubre, we use this approach, because it is safe considering the low risk it involves of injuring the paths of the facial nerve, which occurs more frequently in other approaches. In addition, we obtain a better surgical field and with it a better placement of the osteosynthesis material which results in a shorter surgical time and a lower risk of failure of the placement of the osteosynthesis material. On the other hand, aesthetics is greatly benefited with this approach due to the fact that it is a very small incision and in a discreet anatomical region that is hidden the majority of times or is almost imperceptible.

\section{References}

1. Parihar VS, Bandyopadhyay TK, Chattopadhyay PK, Jacob SM (2019) Retromandibular transparotid approach compared with transmasseteric anterior parotid approach for the management of fractures of the mandibular condylar process: a prospective randomized study. Br J Oral Maxillofac Surg 57: 880-885.

2. Fonseca RJ, Barber HD, Powers MP, Frost DE (2013) Oral and Maxillofacial Trauma. Fonseca RJ, Walker RV (eds) $4^{\text {th }}$ edition, Elsevier Health Sciences, North Carolina, USA. 
3. Hinds EC, Girotti WJ (1967) Vertical subcondylar osteotomy: a reappraisal. Oral Surg Oral Med Oral Pathol 24: 164-170.

4. Ellis E (2012) Surgical approaches to the facial massif. $2^{\text {nd }}$ edition, Caracas, Amolca, 154: 186.

5. Flores JR, Jubilla M, Díaz RG, Rodríguez IZ, Aniceto GS, et al. (2011) Transparotid approach for open reduction of subcondylar fractures. Surgical technique and analysis of its complications. Spanish Journal of Oral and Maxillofacial Surgery 33: 9-14.

6. Bhutia O, Kumar L, Jose A, Roychoudhury A, Trikha A (2014) Evaluation of facial nerve following open reduction and internal fixation of subcondylar fracture through retromandibular transparotid approach. Br J Oral Maxillofac Surg 52: 236-240.
7. Yang L, Patil PM (2012) The retromandibular transparotid approach to mandibular subcondylar fractures. Int J Oral Maxillofac Surg 41: 494-499.

8. Tomar K (2017) Efficacy of the Retroparotid Trans-Masseteric Approach via Retromandibular Incision in ORIF of Subcondylar Fractures: Our Institution Experience. J Maxillofac Oral Surg 17: 332338.

9. Hwang NH, Lee YH, You HJ, Yoon ES, Kim DW (2016) Endoscopeassisted transoral fixation of mandibular condyle fractures: submandibular versus transoral endoscopic approach. J Craniofac Surg 27: 1170-1174. 